\title{
2007/88
}

On the intensity of downside risk aversion

David Crainich and Louis Eeckhoudt 


\title{
CORE DISCUSSION PAPER
}

$2007 / 88$

\section{On the intensity of downside risk aversion}

\section{David CRAINICH ${ }^{1}$ and Louis EECKHOUDT ${ }^{2}$}

November 2007

\begin{abstract}
The degree of downside risk aversion (or equivalently prudence) is so far usually measured by $\frac{-U^{\prime \prime \prime}}{U^{\prime \prime}}$. We propose here another measure, $\frac{U^{\prime \prime \prime}}{U^{\prime}}$, which has interesting properties, different from those related to $\frac{-U^{\prime \prime \prime}}{U^{\prime \prime}}$. It also appears that the two measures are not mutually exclusive. Instead, they seem to be rather complementary as shown through an economic application.
\end{abstract}

Keywords: downside risk aversion, prudence, local and global properties.

JEL Classification: D81

${ }^{1}$ CNRS and LEM, Catholic University of Lille, France.

2 IESEG/LEM, Catholic University of Lille, France and CORE, Université catholique de Louvain, Belgium.

The authors thank Christian Gollier, Patrick Roger, Harris Schlesinger, Fred Schroyen and Arthur Snow for very helpful comments and suggestions.

This paper presents research results of the Belgian Program on Interuniversity Poles of Attraction initiated by the Belgian State, Prime Minister's Office, Science Policy Programming. The scientific responsibility is assumed by the authors. 


\section{Introduction}

It is now common knowledge that in the expected utility model risk aversion is expressed by a negative second derivative of the utility function $\left(U^{\prime \prime}<0\right)$ while its intensity is measured by $\frac{-U^{\prime \prime}}{U^{\prime}}$, the degree of absolute risk aversion (Arrow, 1965; Pratt, 1964).

For downside risk aversion (henceforth D.R.A.), matters are not quite so obvious. There is unanimity to define D.R.A. in the expected utility model by a positive third derivative of the utility function $\left(U^{\prime \prime \prime}>0\right)$. However as far as its intensity is concerned, unanimity breaks down. It is true that most economists suggest to measure the intensity of downside risk aversion only by $\frac{-U^{\prime \prime \prime}}{U^{\prime \prime}}$. This choice is rationalized by the fact the downside risk aversion and prudence are the same thing (both are defined by $U^{\prime \prime \prime}>0$ ) while Kimball (1990) has convincingly argued in favor of $\frac{-U^{\prime \prime \prime}}{U^{\prime \prime}}$ as a measure of the prudence motive. While $\frac{-U^{\prime \prime \prime}}{U^{\prime \prime}}$ and its properties (e.g. "decreasing prudence") appeared to be very useful in many contexts so that $\frac{-U^{\prime \prime \prime}}{U^{\prime \prime}}$ was considered as the measure of downside risk aversion, two recent papers - to the best of our knowledge - questioned this point of view. The first one by Keenan and Snow (2002) is based on the notion of compensated increases in risk as defined by Diamond and Stiglitz (1974). In that context they obtain that the index of downside risk aversion is made of two terms, one of them ${ }^{1}$ being equal to $\frac{U^{\prime \prime \prime}}{U^{\prime}}$. More recently, Modica and Scarsini (2006), starting from Ross' notion of stronger risk aversion (1981) obtained also that the degree of local downside risk aversion should be measured by $\frac{U^{\prime \prime \prime}}{U^{\prime}}$. However they observed thet "this coefficient does not allow to translate a local comparison into a global comparison" (see page 270 in their paper) contrarily to what happens for the Arrow-Pratt coefficient of the absolute risk aversion.

The purpose of the present paper is twofold. First we want to derive $\frac{U^{\prime \prime \prime}}{U^{\prime}}$ as a measure of D.R.A. intensity directly from an approach à la ArrowPratt $^{2}$. Beside we will show that this local measure does have interesting global properties and that it can give rise to relevant economic interpretations.

More specifically the paper is organized as follows. In section 2 the main result is presented and an intuitive approach is offered in section 3 . The global properties of the local measure $\frac{U^{\prime \prime \prime}}{U^{\prime}}$ are discussed in section 4 while an economic interpretation of the new measure of D.R.A. is given in section 5 .

\footnotetext{
${ }^{1}$ The other is related to the degree of absolute risk aversion. See their equation (7) and its discussion.

${ }^{2}$ Notice that the two papers just mentioned didn't use the Arrow-Pratt approach.
} 
In the conclusion we discuss the complementarity between the usual measure and the new one.

\section{The main result}

From Menezes, Geiss and Tressler (1980) and its presentation in Eeckhoudt and Schlesinger (2006) (from now on E-S) we know that in the expected utility model:

$$
\frac{1}{2} U(x-k)+\frac{1}{2} E U(x+\widetilde{\epsilon})>\frac{1}{2} U(x)+\frac{1}{2} E U(x-k+\widetilde{\epsilon}) \Longleftrightarrow U^{\prime \prime \prime}>0
$$

where $x$ is initial wealth, $k$ is a positive constant and $\widetilde{\epsilon}$ a zero mean risk.

In E-S's terminology the left hand side (LHS) of (1) is preferred to the right hand side (RHS) because for the LHS term, the pains $(-k$ and $\widetilde{\epsilon})$ are "better apportioned" than for the RHS term. More precisely, on the LHS, the pains are disaggregated while it is not the case on the RHS where they are concentrated on a single state of nature.

Since with $U^{\prime \prime \prime}>0$ welfare is higher on the LHS of (1) we can then search for the amount of money that compensates for the misallocation of the pains on the RHS of (1).

If this amount of money - denoted $m$ - is received in the best state $(x)$ on the RHS of (1), it is the solution of ${ }^{3}$ :

$$
\frac{1}{2} U(x-k)+\frac{1}{2} E U(x+\widetilde{\epsilon})=\frac{1}{2} U(x+m)+\frac{1}{2} E U(x-k+\widetilde{\epsilon})
$$

For small risks $\widetilde{\epsilon}$ and applying a second order approximation à la ArrowPratt we obtain:

$$
\frac{1}{2} U(x-k)+\frac{1}{2}\left[U(x)+\frac{\sigma^{2}}{2} U^{\prime \prime}(x)\right] \cong \frac{1}{2} U(x+m)+\frac{1}{2}\left[U(x-k)+\frac{\sigma^{2}}{2} U^{\prime \prime}(x-k)\right]
$$

Multiplying by 2 on each side and simplifying the common term $U(x-k)$, we are left with:

$$
\frac{\sigma^{2}}{2}\left(U^{\prime \prime}(x)-U^{\prime \prime}(x-k)\right) \cong U(x+m)-U(x)
$$

\footnotetext{
${ }^{3}$ Of course many other forms of compensations are possible. They lead basically to the same result after sometimes less transparent developments.
} 
or, after a first order approximation around $x$ :

$$
\frac{\sigma^{2}}{2} k U^{\prime \prime \prime}(x) \cong m U^{\prime}(x)
$$

so that:

$$
m \cong \frac{\sigma^{2}}{2} k \frac{U^{\prime \prime \prime}(x)}{U^{\prime}(x)}
$$

Of course as expected $U^{\prime \prime \prime}(x)>0$ implies $m>0$ and $\frac{U^{\prime \prime \prime}(x)}{U^{\prime}(x)}$ is the strength of the D.R.A. motive which confirms from another point of view the interest of the measure proposed by Modica and Scarsini. Of course, the higher the coefficient of D.R.A. $\left(\frac{U^{\prime \prime \prime}(x)}{U^{\prime}(x)}\right)$ the larger the compensation required to accept a misallocation of the pains.

Many readers may rightly wonder why skewness does not appear in the approximation formula since it involves third order terms ${ }^{4}$. In fact, as shown in the appendix, the expression $\frac{\sigma^{2}}{2} k$ is proportional to the change in skewness induced by the misapportionment of the risk. Keeping this in mind, the approximation of $m$, the cost of the misallocation of the pain, appears to be determined by two factors:

- the importance of the change in skewness (a statistical property).

- the intensity of the D.R.A. coefficient expressed by $\frac{U^{\prime \prime \prime}(x)}{U^{\prime}(x)}$ (a preference property).

Seen from this point of view, our result about the approximation of $m$ exactly parallels the approximation of the risk premium in Arrow-Pratt.

\section{An intuitive approach}

In a now much neglected paper, Friedman and Savage (1948) had defined the "utility premium" denoted $R$ by:

$$
R=U(x)-E U(x+\widetilde{\epsilon})
$$

For concave $U, R$ is positive and measures the pain inflicted by the presence of a zero mean risk. Using the standard approximation for small risks:

$$
R \approx \frac{\sigma^{2}}{2}\left(-U^{\prime \prime}(x)\right)
$$

\footnotetext{
${ }^{4}$ The relationship between skewness and the third derivative of $U$ has been discussed in many papers. A list of such papers can be found in Scott and Horvath (1980).
} 
As is well known, the risk premium (which is an amount of money, denoted $\pi$ ) is given by:

$$
\pi \approx \frac{\sigma^{2}}{2}\left(\frac{-U^{\prime \prime}(x)}{U^{\prime}(x)}\right)
$$

or

$$
\pi \approx \frac{R}{U^{\prime}(x)}
$$

The division of $R$ by $U^{\prime}(x)$ to generate $\pi$ can then be easily understood: its purpose is to convert into money the pain due to risk $\widetilde{\epsilon}$ and the conversion factor is the marginal utility of money. The higher $U^{\prime}(x)$, the lower the amount of money a decision maker is willing to give up in order to eliminate a given pain ${ }^{5}$.

In fact a similar approach can be used to justify the use of $\frac{U^{\prime \prime \prime}}{U^{\prime}}$ as a measure of the intensity of D.R.A. The pain due to a misapportionment of a risk $\widetilde{\epsilon}(\operatorname{denoted} S)$ is defined by:

$$
S=\frac{1}{2} U(x-k)+\frac{1}{2} E U(x+\widetilde{\epsilon})-\frac{1}{2} U(x)-\frac{1}{2} E U(x-k+\widetilde{\epsilon})
$$

For a small risk $\widetilde{\epsilon}$, one has:

$$
S \approx \frac{1}{2} \frac{\sigma^{2}}{2}\left[U^{\prime \prime}(x)-U^{\prime \prime}(x-k)\right]
$$

and then for small $k$ :

$$
S \approx \frac{\sigma^{2}}{4} k U^{\prime \prime \prime}(x)
$$

To transform this pain into a money equivalent we have to divide by $U^{\prime}(x)$ (as for the risk premium) and we then obtain an expression equal to:

$$
\frac{\sigma^{2}}{4} k \frac{U^{\prime \prime \prime}(x)}{U^{\prime}(x)}
$$

which is simply $\frac{m}{2}$ (see eq.5).

This result not only confirms $\frac{U^{\prime \prime \prime}}{U^{\prime}}$ as a measure of downside risk aversion. It also establishes the close link with the well accepted measure of

\footnotetext{
${ }^{5}$ Notice that a similar approach is used by Foncel and Treich (2005) to analyse the concept of fear of ruin developed by Aumann and Kurz (1977) and which is equal to $\frac{U}{U^{\prime}}$. Besides, in a recent and innovative paper based on comparative statics properties, Jindapon and Neilson (2007) present the Ross' equivalent of prudence through the division of $U^{\prime \prime \prime}$ by $U^{\prime}$.
} 
absolute risk aversion $\left(\frac{-U^{\prime \prime}}{U^{\prime}}\right)$ through the concept of utility premium and its conversion in monetary terms.

\section{A global property}

As is well known the Arrow-Pratt local index of absolute risk aversion has a nice global property, namely that if $V(x)=s(U(x))$ with $s^{\prime}>0$ and $s^{\prime \prime}<0$ then $V$ has a higher degree of absolute risk aversion than $U$ at any $x$. Hence it is natural to wonder if a local measure of the intensity of D.R.A. posesses a similar property.

For Kimball's local measure $\left(\frac{-U^{\prime \prime \prime}}{U^{\prime \prime}}\right)$ it is already known that global properties are not easily obtained. As shown in Eeckhoudt and Schlesinger $(1994)^{6}$, if $s^{\prime}>0$ and $s^{\prime \prime}<0$ prevail so that $V$ is more risk averse than $U$, the additional condition $s^{\prime \prime \prime}>0$ implies more D.R.A. only if quite demanding restrictions are imposed on the utility function.

When $\frac{U^{\prime \prime \prime}}{U^{\prime}}$ is used as the local measure of the intensity of D.R.A., matters are much easier.

Indeed let $V(x)=s(U(x))$ with $s^{\prime}>0, s^{\prime \prime}<0$ and $s^{\prime \prime \prime}>0$. Then one easily obtains:

$$
\frac{V^{\prime \prime \prime}}{V^{\prime}}=\frac{s^{\prime \prime \prime}}{s^{\prime}}\left(U^{\prime}\right)^{2}+3 \frac{s^{\prime \prime}}{s^{\prime}} U^{\prime \prime}+\frac{U^{\prime \prime \prime}}{U^{\prime}}
$$

Since $3 \frac{s^{\prime \prime}}{s^{\prime}} U^{\prime \prime}$ is positive for risk averse decision makers, the joint conditions $s^{\prime \prime}<0$ and $s^{\prime \prime \prime}>0$ are sufficient ${ }^{7}$ to imply that the utility function $V$ simultaneously displays more risk aversion and more downside risk aversion than $U$ at any $x$.

It thus appears that the local measure $\frac{U^{\prime \prime \prime}}{U^{\prime}}$ has global properties that are much more intuitive than those attached to $\frac{-U^{\prime \prime \prime}}{U^{\prime \prime}}$.

\section{$5 \quad$ An economic application}

Following Kimball (1990) it is now well known that the local coefficient of prudence $\frac{-U^{\prime \prime \prime}}{U^{\prime \prime}}$ can be used to characterize precautionary savings motive. When $\frac{-U^{\prime \prime \prime}}{U^{\prime \prime}}$ increases caeteris paribus the decision maker increases the quantity of precautionary savings he wants to build up in order to better face

\footnotetext{
${ }^{6}$ See especially their corollary 3.4 .

${ }^{7} s^{\prime \prime \prime}>0$ is sufficient but not necessary to yield the result.
} 
future income risk. Hence a link is created between $\frac{-U^{\prime \prime \prime}}{U^{\prime \prime}}$ and a quantity demanded (in this case that of precautionary savings).

We now show that the other measure of the prudence motive $\left(\frac{U^{\prime \prime \prime}}{U^{\prime}}\right)$ is linked to an equilibrium price (instead of a quantity demanded). In order to maintain the link with the savings literature consider a very simple economy with no physical investment and where future consumption $\left(\widehat{c}_{2}\right)$ is random because future income $y_{2}$ is risky. In this economy the representative consumer maximizes:

$$
U\left(c_{1}\right)+\frac{1}{1+\theta} E\left[U\left(\left(y_{1}-c_{1}\right)(1+r)+\widehat{y}_{2}\right)\right]
$$

where $\theta$ is the discount rate applied to the next period utility and $r$ is the safe return on savings. As usual, $c_{1}$ and $y_{1}$ represent respectively current consumption and current income.

The optimality condition is written:

$$
U^{\prime}\left(c_{1}\right)-\frac{(1+r)}{1+\theta} E\left[U^{\prime}\left(\left(y_{1}-c_{1}\right)(1+r)+\widehat{y}_{2}\right)\right]=0
$$

If there is no investment, in equilibrium $c_{1}=y_{1}$ and the optimality condition becomes then ${ }^{8}$ :

$$
U^{\prime}\left(c_{1}\right)-\frac{(1+r)}{1+\theta} E\left[U^{\prime}\left(\widehat{y}_{2}\right)\right]=0
$$

When $\widehat{y}_{2}$ is non random and if $y_{1}=y_{2}$ the equilibrium rate of return on saving is equal to the discount rate $\theta$.

Should $\widehat{y}_{2}$ become random with $E\left(\widehat{y}_{2}\right)=y_{1}$ and if utility is quadratic, the uncertainty on $\widehat{y}_{2}$ has no effect on the equilibrium rate $r$ essentially because with a quadratic utility $U^{\prime \prime \prime}=0$. When $U$ is quadratic future income uncertainty does not generate an additional savings demand so that the equilibrium rate of interest is not affected.

In order to isolate the effect of a D.R.A. on $r$, consider now that $U$ is cubic in consumption levels such that $U^{\prime}>0, U^{\prime \prime}<0$ and $U^{\prime \prime \prime}>0^{9}$. In this case we have:

$$
E\left[U^{\prime}\left(\widehat{y}_{2}\right)\right]=U^{\prime}\left(E\left(\widehat{y}_{2}\right)\right)+\frac{\sigma^{2}}{2} U^{\prime \prime \prime}\left(E\left(\widehat{y}_{2}\right)\right)
$$

\footnotetext{
${ }^{8}$ Condition (9) is one of the two equilibrium conditions discussed in Barsky (1989). Barsky's model is more general than the present one since it allows for the existence of a safe and a risky asset.

${ }^{9}$ By limiting ourselves to cubic utility we do not introduce effects of higher orders such as temperance or edginess that affect also the equilibrium value of $r$.
} 
so that for $y_{1}=c_{1}$ and $E\left[\widehat{y}_{2}\right]=y_{1}$, (9) becomes:

$$
U^{\prime}\left(y_{1}\right)-\frac{(1+r)}{1+\theta} U^{\prime}\left(y_{1}\right)\left[1+\frac{\sigma^{2}}{2} \frac{U^{\prime \prime \prime}\left(y_{1}\right)}{U^{\prime}\left(y_{1}\right)}\right]=0
$$

Hence in equilibrium one now has:

$$
(1+r)=\frac{1+\theta}{1+\frac{\sigma^{2}}{2} \frac{U^{\prime \prime \prime}\left(y_{1}\right)}{U^{\prime}\left(y_{1}\right)}}
$$

The presence of future income uncertainty depresses the equilibrium rate of interest and it becomes lower than $\theta$. This result is in accordance with intuition since the increased saving generated by future income risk has to remain equal in equilibrium to the fixed zero level of investment and this can be achieved only by a fall in $r$. What equation (11) reveals further is that the impact on equilibrium $r$ of future income uncertainty (expressed by $\sigma^{2}$, the variance of $\widehat{y}_{2}$ ) is related to the size of $\frac{U^{\prime \prime \prime}}{U^{\prime}}$, the alternative measure of D.R.A. intensity. When $\frac{U^{\prime \prime \prime}}{U^{\prime}}$ increases, caeteris paribus, the equilibrium rate of return on savings falls.

This result clarifies the respective roles of the two "competing" measures of downside risk aversion: while $\frac{-U^{\prime \prime \prime}}{U^{\prime \prime}}$ is useful to interpret changes in (market) quantities, $\frac{U^{\prime \prime \prime}}{U^{\prime}}$ is related to the induced change in market equilibrium price. This result should not be surprising since as shown in section 3 the division of the pain due to misapportionment by $U^{\prime}$ leads to a money equivalent which is indeed close to the notion of price.

\section{Conclusion}

The local measure of prudence (or downside risk aversion) suggested by Kimball, $\frac{-U^{\prime \prime \prime}}{U^{\prime \prime}}$, has been very useful to develop new tools for the economic analysis under risk.

In this paper we have proposed another local measure, $\frac{U^{\prime \prime \prime}}{U^{\prime}}$, which turns out to also have interesting properties. First, it is related quite directly to the standard concept of absolute risk aversion, as shown in sections 2 and 3. Besides it has a global property that is a rather natural extension of the one obtained for the coefficient of absolute risk aversion.

All this doesn't mean that one measure dominates the other. Instead they are more complementary than competitors. For instance, as indicated in section $5, \frac{-U^{\prime \prime \prime}}{U^{\prime \prime}}$ explains changes in quantities demanded or supplied while $\frac{U^{\prime \prime \prime}}{U^{\prime}}$ is more appropriate to discuss changes in equilibrium prices. 


\section{Appendix}

Consider first the lottery $\widetilde{z}$ on the LHS of (1) i.e.

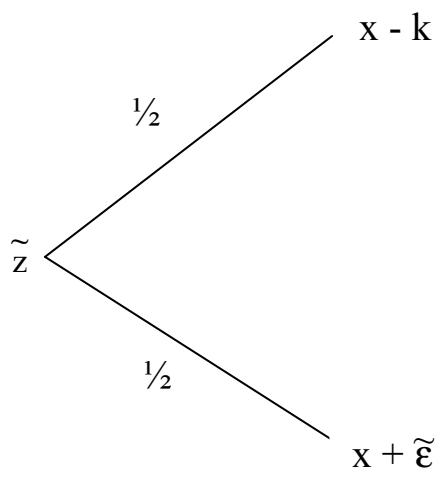

Lottery $\widetilde{z}$

where $E(\widetilde{\varepsilon})=0$. Denoting by $\sigma^{2}$ and $\mu_{3}$ respectively the variance and the skewness ${ }^{10}$ of $\widetilde{\varepsilon}$, we find after easy manipulations:

$$
\begin{aligned}
E(\widetilde{z}) & =x-\frac{k}{2} \\
\operatorname{var}(\widetilde{z}) & =\frac{\sigma^{2}}{2}+\frac{k^{2}}{4} \\
\operatorname{skewness}(\widetilde{z}) & =\frac{1}{2} \mu_{3}+\frac{3}{4} k \sigma^{2}
\end{aligned}
$$

Consider now the lottery $\widetilde{y}$ on the RHS of (1) i.e.

\footnotetext{
${ }^{10}$ For a random variable $\widetilde{x}: \mu_{3}=E(\widetilde{x}-\mu)^{3}$
} 


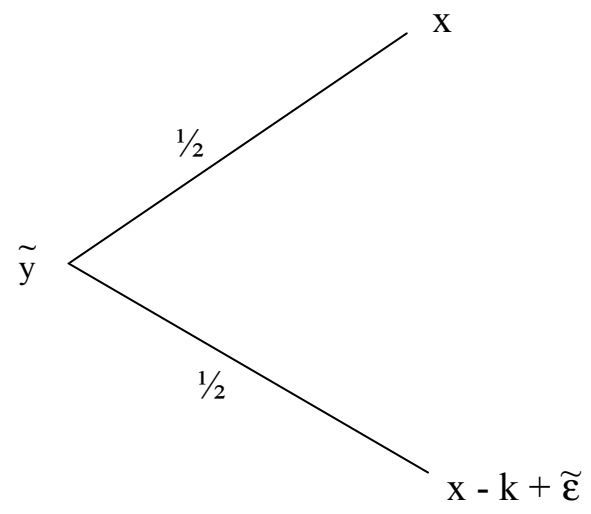

Lottery $\widetilde{y}$

One easily obtains:

$$
\begin{aligned}
E(\widetilde{y}) & =x-\frac{k}{2} \\
\operatorname{var}(\widetilde{y}) & =\frac{\sigma^{2}}{2}+\frac{k^{2}}{4} \\
\operatorname{skewness}(\widetilde{y}) & =\frac{1}{2} \mu_{3}-\frac{3}{4} \sigma^{2} k
\end{aligned}
$$

Of course the lotteries $\widetilde{z}$ and $\widetilde{y}$ have the same mean and variance. Yet they do not have the same skewness and going from $\widetilde{y}$ to $\widetilde{z}$ increases skewness by $\frac{3}{2} \sigma^{2} k$. Such an increase in skewness is positively appreciated by individuals with $U^{\prime \prime \prime}>0$ and we observe that in (5) the degree of D.R.A. $\left(\frac{U^{\prime \prime \prime}(x)}{U^{\prime}(x)}\right)$ is multiplied by a term that is proportional to the change in skewness to obtain $m$. Hence skewness is implicitly present in (5).

\section{References}

Aumann, Robert and Mordecai Kurz. (1977). "Power and taxes," Econometrica 45(5), 1137-1161.

Arrow, Kenneth (1965) "Aspects of the theory of risk-bearing", Helsinki, Yrjš Jahnsson Foundation.

Barsky, Robert (1989), "Why don't the prices of stocks and bonds move together?, American Economic Review 79, 1132-1145.

Diamond, Peter and Joseph Stiglitz. (1974). "Increases in risk and in risk aversion," Journal of Economic Theory 8(3), 335-360. 
Eeckhoudt, Louis and Harris Schlesinger. (2006). "Putting risk in its proper place," American Economic Review 96(1), 280-289.

Eeckhoudt, Louis and Harris Schlesinger. (1994). "A precautionary tale of risk aversion and prudence," in Models and experiments in risk and rationality, B. Munier and M. Machina, eds. Kluwer Academic Publishers, 75-90.

Foncel, Jérôme and Nicolas Treich. (2005). "Fear of ruin," The Journal of Risk and Uncertainty 31(3), 289-300.

Friedman, Milton, Leonard Savage. (1948). "The utility analysis of choices involving risk," Journal of Political Economy 56(4), 279-304.

Jindapon, Paan and William Neilson. (2007). "Higher-order generalizations of Arrow-Pratt and Ross risk aversion: A comparative statics approach," Journal of Economic Theory 136(1), 719-728

Keenan Donald and Arthur Snow. (2005). "Greater downside risk aversion," The Journal of Risk and Uncertainty 24 (3), 267-277.

Kimball, Miles. (1990). "Precautionary saving in the small and in the large," Econometrica 58(1), 53-73.

Modica, Salvatore and Marco Scarsini. (2005). "A note on comparative downside risk aversion," Journal of Economic Theory 122, 267-271

Menezes Carmen, C. Geiss and John Tressler. (1980). "Increasing downside risk," American Economic Review 70(5), 921-932.

Pratt, John. (1964). "Risk aversion in the small and in the large," Econometrica 32, 122-136.

Ross, Stephen. (1981). "Some stronger measures of risk aversion in the small and the large with applications," Econometrica 49(3), 621-638.

Scott, Robert and Philip Horvath. (1980). "On the direction of preference for moments of higher order than the variance," The Journal of Finance 35(4), 915-919. 